\title{
Modeling resistance instabilities of set and reset states in phase change memory with Ge-rich $\mathrm{GeSbTe}$
}

\author{
N. Ciocchini, Student Member, IEEE, E. Palumbo, M. Borghi, P. Zuliani, R. Annunziata, D. Ielmini, Senior \\ Member, IEEE
}

\begin{abstract}
To satisfy the growing market demand for embedded non-volatile memory (eNVM), alternative solutions to Flash technology are currently under investigation. Among these, phase change memory (PCM) is attracting strong interest due to the low cost of integration with the CMOS front-end and good scalability. Embedded PCM (ePCM), however, must feature high reliability during both packaging and functional stages. This work studies reliability of PCM based on Ge-rich GeSbTe, providing evidence for resistance drift and decay in both the reset and set states. Set state instability is attributed to grain-boundary relaxation and grain growth. A unified model is presented, capable of predicting the reliability of set/reset states at elevated temperature.
\end{abstract}

Keywords: Phase change memory, embedded memory, set drift, soldering, reliability modeling

\section{INTRODUCTION}

Phase change memory (PCM) is one of the main emerging memory technology, featuring nanosecond phase switching [1], low programming power [2] and good reliability and scalability. PCM properties can be tailored by material and cell engineering thus allowing for multiple applications, such as stand-alone nonvolatile memory [3], storage class memory (SCM) [4] and embedded memories in microcontrollers and smart cards [5]. Embedded phase change memory (ePCM) can be scaled to future lithographic nodes and is compatible with high-k metal gate logic, given its integration in the back-end of the line (BEOL) technology. On the other hand, ePCM should also feature data retention at high temperature, e.g., $150^{\circ} \mathrm{C}$ in automotive applications, and during packaging, where the temperatures can rise above $250^{\circ} \mathrm{C}$ for few minutes. To satisfy these tough reliability constraints, new chalcogenide materials with improved crystallization temperature were proposed, namely Ge-rich GeSbTe [6]-[8], C-doped GeTe [9] and $\mathrm{SiO}_{2}$ doped GeTe [10].

In this work, we address data retention in ePCM with Gerich GeSbTe, where we evidence a resistance drift and decay for both the set (poly-crystalline) and the reset (amorphous) states. These effects are modeled by structural relaxation

N. Ciocchini and D. Ielmini are with the Dipartimento di Elettronica e Informazione and Italian Universities Nanoelectronics Team (IU.NET), Politecnico di Milano, piazza L. da Vinci 32, 20133 Milano, Italy. E-mail daniele.ielmini@polimi.it

E. Palumbo, M. Borghi, P. Zuliani and R. Annunziata are with STMicroelectronics, Agrate Brianza, Italy.

Work at Politecnico di Milano was supported in part by Fondazione Cariplo under Grant 2010-0500.

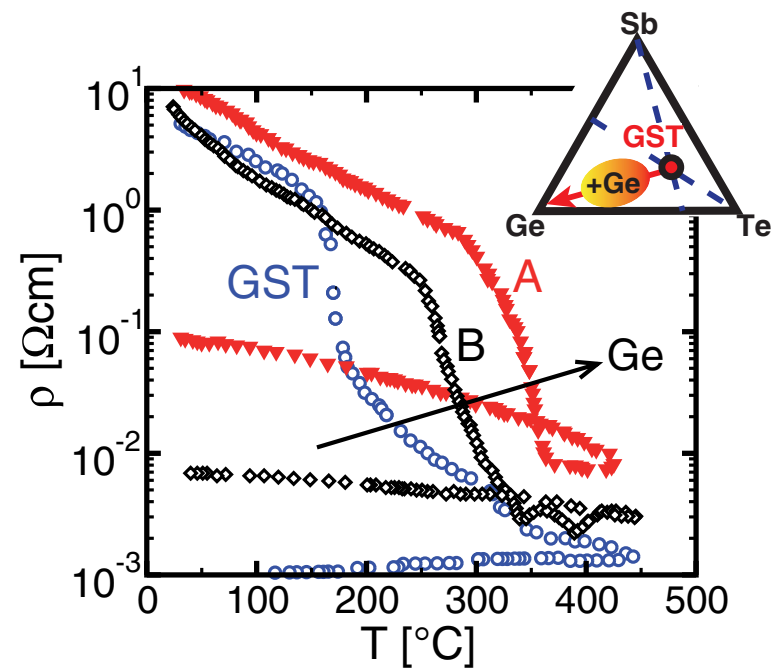

Fig. 1. Measured resistivity-temperature characteristic for different chalcogenide materials $\left(T\right.$-ramp rate $\left.10^{\circ} \mathrm{C} / \mathrm{min}\right)$. Note how the crystallization temperature $T_{X}$ increases by increasing the Ge $\%$ from GST to alloy A.

and crystallization of amorphous chalcogenide phase, possibly residing at the grain boundaries in the set state [8]. We thus develop a unified model, capable of a physical description and accurate prediction of ePCM data retention for variable annealing conditions and programmed states. The present work extends the results of Ref. [8] by showing new experimental data and a more detailed explanation of the set/reset instabilities model. In particular, we added the comparison between PCM device with conventional active material and Ge-rich GeSb-Te (Figs. 2-5) and we validated our physical interpretation by measuring the activation energy for conduction $E_{C}$ in both the drift and decay regimes (Fig. 10). The onset of resistance decay after drift has been also modeled in detail in Fig. 15, allowing for an accurate prediction of resistance window as a function of operation temperature.

\section{Material AND CELl ENGINEeRing}

Fig. 1 shows the measured resistivity as a function of temperature $(T)$ during a ramped- $T$ annealing experiment with heating rate of $10^{\circ} \mathrm{C} \mathrm{min}-1$. The figure compares GST $\left(\mathrm{Ge}_{2} \mathrm{Sb}_{2} \mathrm{Te}_{5}\right)$ with two Ge-rich GeSbTe alloys, namely material B and A with increasing Ge content (see inset). The resistivity first decreases due to the temperature-activated conduction in the amorphous phase, then sharply drops as a 


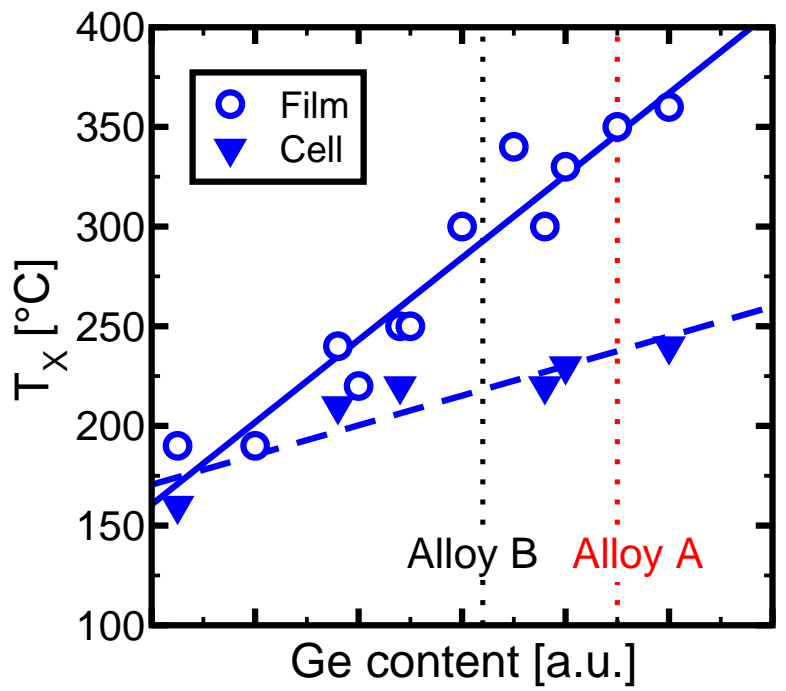

Fig. 2. Measured $T_{X}$ as a function of $\mathrm{Ge} \% . T_{X}$ is smaller after integration in the PCM cell, due both to the different experimental technique employed ( $T$-ramp $10^{\circ} \mathrm{C} / \mathrm{min}$ for the film and 1 hour bake for the cell) and possible local composition variation upon set/reset pulses on the PCM cell.

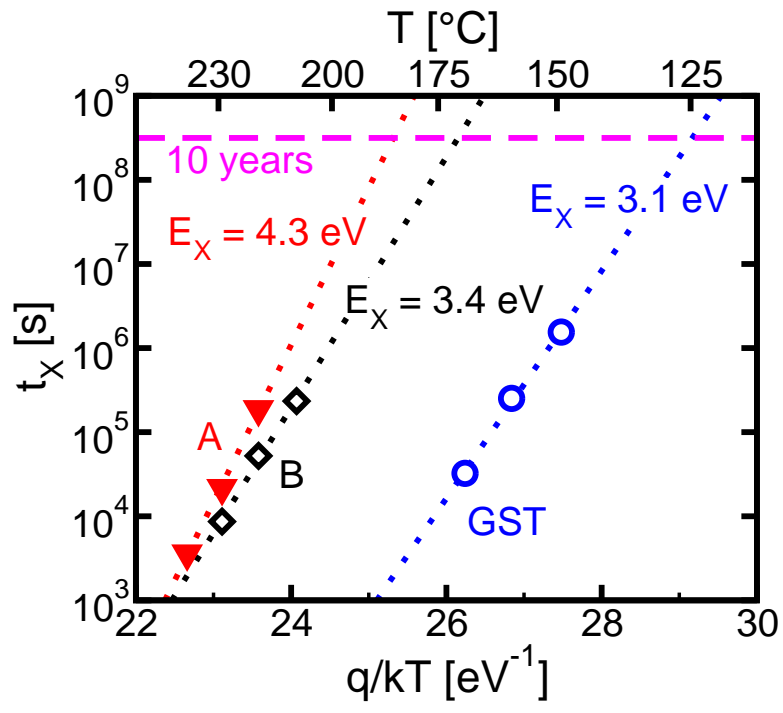

Fig. 3. Arrhenius plot of $t_{X}$ for PCM with GST, alloy A and B. The activation energy increases and the stable $T$ range at 10 years increases from GST to alloy A.

result of crystallization. The crystallization temperature $T_{X}$ can be defined in correspondence of the maximum slope in the $R-T$ curves: $T_{X}$ increases from about $150^{\circ} \mathrm{C}$ for GST to about $350^{\circ} \mathrm{C}$ for alloy A. The same measurement was repeated on many $\mathrm{Ge}-\mathrm{Sb}$-Te compositions with different $\mathrm{Ge}$ content. Experimental results in Fig. 2 clearly demonstrate the linear increase of $T_{X}$ with $\mathrm{Ge} \%$ in both thin film samples and PCM cells. The increase of $T_{X}$ at higher Ge content can be attributed to the increase of tetrahedral Ge-Ge bonds [11], which stabilize the amorphous structure with respect to crystallization. PCM devices with $90 \mathrm{~nm}$ mushroom-type structure were fabricated using GST, alloy A or alloy B as active material [8]. Fig. 3 shows the Arrhenius plot of the crystallization time, defined by the drop of resistance below $R=100 \mathrm{k} \Omega$ of reset-state PCM devices during constant- $T$

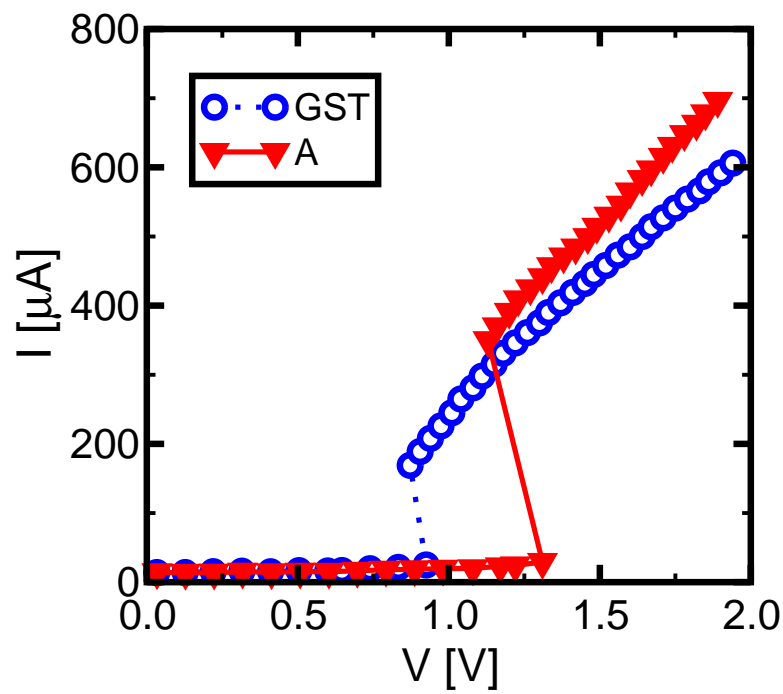

Fig. 4. IV characteristics of the ePCM with alloy A and GST as active layers. The higher $V_{T}$ of alloy A allows better read-disturb immunity.

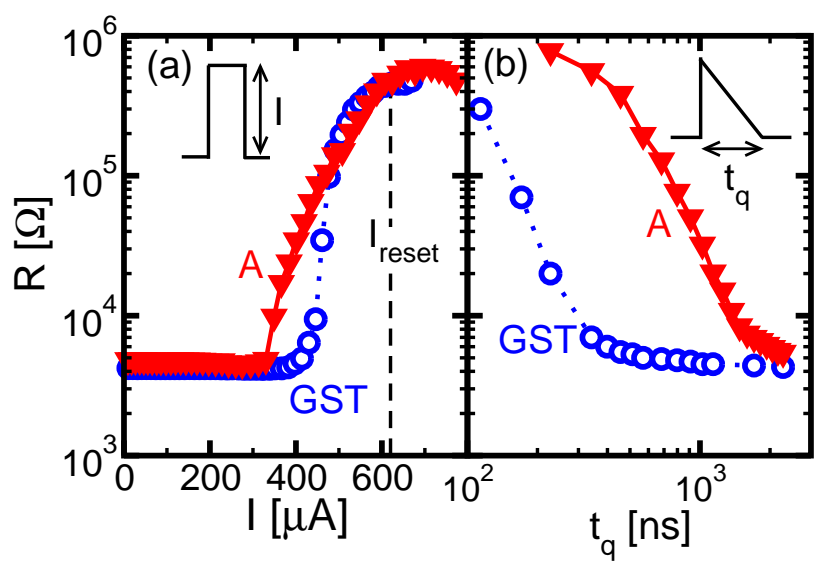

Fig. 5. Measured reset (a) and set (b) programming characteristics for alloy A and GST. In (a), resistance was measured for increasing current (inset), showing reset at about $600 \mu \mathrm{A}$ for both materials. In (b), a triangular pulse with quenching time $t_{q}$ was applied. Alloy A reaches set state in about $2 \mu \mathrm{s}$.

annealing [8]. The data satisfy the Arrhenius law given by:

$$
t_{X}=\tau_{0 X} e^{\frac{E_{X}}{k_{B} T}}
$$

where $\tau_{0 X}$ is a time constant, $k_{B}$ is the Boltzmann constant and $E_{X}$ is the activation energy for crystallization. Using Eq. (1) to fit $t_{X}$ in Fig. 3 we obtain $E_{X}=3.1 \mathrm{eV}$ for GST, $3.4 \mathrm{eV}$ for alloy B and $4.3 \mathrm{eV}$ for alloy A. From these results, both $t_{X}$ at a given $T$ and $E_{X}$ increase monotonically at increasing Ge content, in agreement with [6], [12]. This is a key guideline for the optimization of the PCM material for high temperature retention. In the following, we will restrict our attention to alloy A and to the reference GST material.

Fig. 4 shows the I-V characteristics of ePCM with alloy A and GST. Threshold voltage $V_{T}$ for alloy A is higher than GST, which allows better stability over read disturb [13]. Fig. 5a shows the programming characteristics of PCM devices with GST and alloy A, namely the PCM resistance $R$ as a function of the current of the applied square pulse. The reset transition appears as the rise of resistance in correspondence of a reset current $I_{\text {reset }}$ of about $600 \mu \mathrm{A}$ for both GST and alloy A. This 

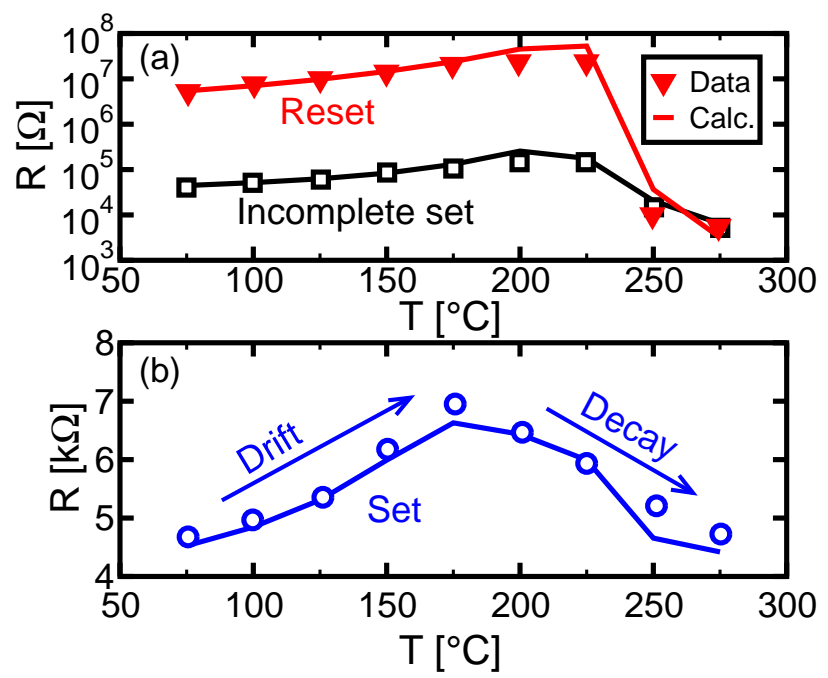

Fig. 6. Measured resistance for increasing annealing temperature for reset, incomplete set (a) and set state in (b). Each annealing step lasted 1 hour, while the resistance is measured at room temperature. All the states show an increase of $R$ (drift regime) followed by a drop of $R$ (decay regime).

indicates that $I_{\text {reset }}$ is negligibly changed by increasing the Ge content, thus allowing for low power consumption in embedded memories. Fig. 5b shows the set transition characteristics, where $R$ is monitored after the application of a triangular pulse with current $I_{\text {reset }}$ and with increasing quenching time $t_{q}$ (see inset). $R$ decreases for increasing $t_{q}$, indicating the transition from the amorphous phase, obtained at fast quenching from the molten phase, to the poly-crystalline phase, originating from the regrowth of ordered grains at the boundary between the crystalline GST and the molten phase [14]. To achieve full crystallization, $t_{q}$ is around $500 \mathrm{~ns}$ for GST and $2 \mu \mathrm{s}$ for alloy A. Note that Fig. 5b seems in contradiction with Fig. 1, where the set-state resistance of GST and Ge-rich Ge-Sb-Te differs by an order of magnitude in Fig. 1, while they are almost equal in Fig. 5b. This difference might be attributed to the fact that the PCM device in Fig. 5b was programmed by electrical set/reset processes, which might induce local composition changes in the phase change region above the bottom electrode contact [15]. The longer set transition is the drawback of the increased Ge content, stabilizing the amorphous phase against crystallization as already seen in Figs. 1 and 3. Note, however, that a $2 \mu$ s set transition is much faster than the programming time of floating-gate memories, thus ePCM solution is advantageous in terms of speed for embedded applications.

\section{RELIABILITY CHARACTERISTICS}

To study $R$ stability in PCM devices with alloy A, we performed annealing experiments for $1 \mathrm{~h}$ at constant annealing temperature $T$. Fig. 6 shows the PCM resistance measured at room temperature for reset, incomplete set states (a) and for the set state (b) as a function of $T$. The incomplete set and the set states were obtained with triangular crystallization pulses with $t_{q}=1 \mu \mathrm{s}$ and $3 \mu \mathrm{s}$, respectively. All states in the figure display similar behavior, namely an increase of $R$ in the drift regime, followed by a sharp decrease in the decay regime. GST-based PCM devices programmed in the reset state are

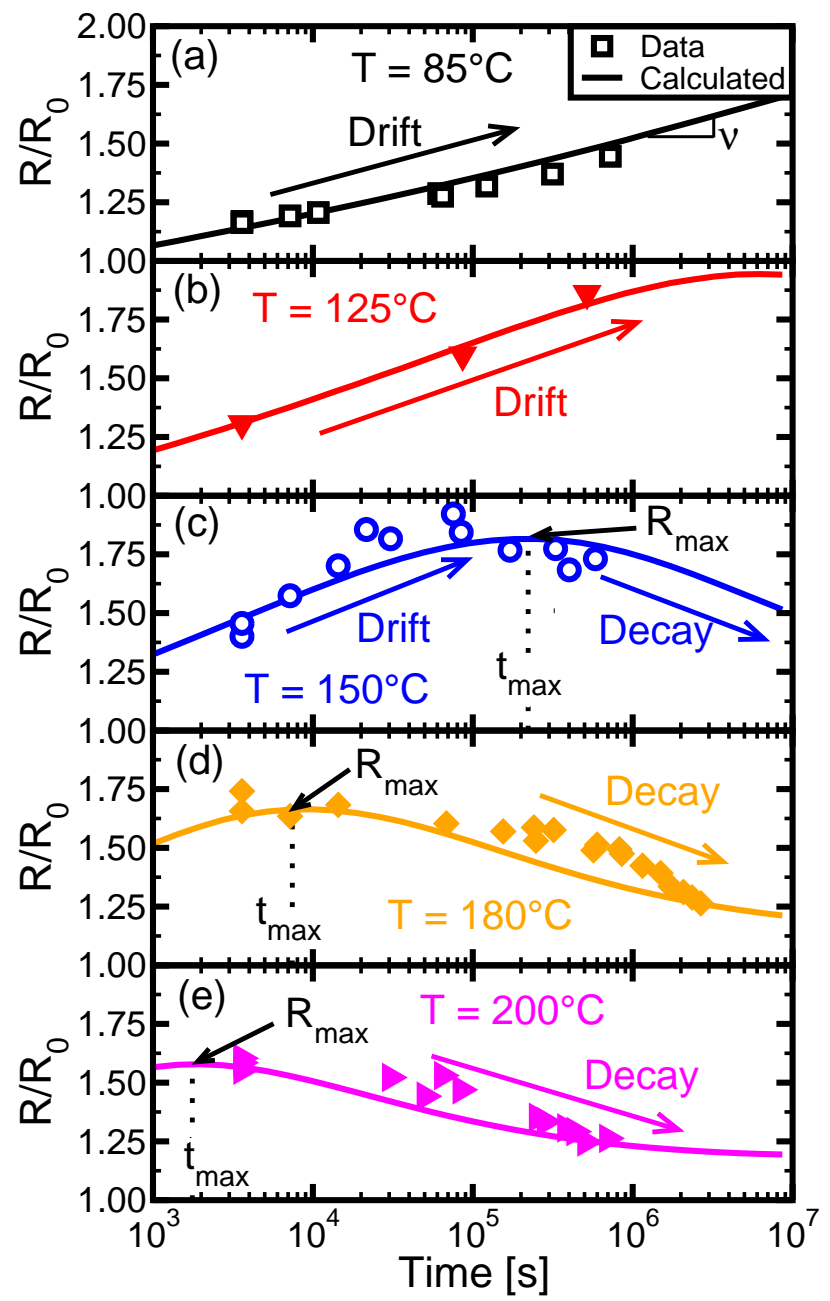

Fig. 7. Measured and calculated $R(t)$ for the set state at annealing temperature of $85^{\circ} \mathrm{C}(\mathrm{a}), 125^{\circ} \mathrm{C}(\mathrm{b}), 150^{\circ} \mathrm{C}(\mathrm{c}), 180^{\circ} \mathrm{C}(\mathrm{d})$ and $200^{\circ} \mathrm{C}(\mathrm{e})$, indicating the $T$-acceleration of drift/decay. $R_{\max }$ and corresponding $t_{\max }$ are taken at the transition between the drift and decay regimes.

known to display drift [16], [17] and resistance decrease due to crystallization. Most recently, drift [5], [8], [10] and resistance decay [8] were reported for Ge-rich GeSbTe in the set state. Note that no set resistance drift was previously reported for conventional GST [18], which is the standard material for stand-alone PCM devices. This suggests a dominant role of Ge in the structural relaxation of chalcogenide materials [19]. Since the drift effect is detrimental on the set side of the reading window, a predictive model for extended reliability of ePCM based on Ge-rich GeSbTe is required. To further analyze the drift and decay regimes of the set-state resistance, Fig. 7 shows $R$ measured at room temperature as a function of annealing time for isothermal conditions at increasing temperature $T=85^{\circ} \mathrm{C}$ (a), $125^{\circ} \mathrm{C}$ (b), $150^{\circ} \mathrm{C}$ (c), $180^{\circ} \mathrm{C}$ (d) and $200^{\circ} \mathrm{C}(\mathrm{e})$. The results clearly evidence the $T$-accelerated nature of drift and decay of resistance in the set state: For relatively-low $T$ (Fig. 7a and b) the resistance evolution is dominated by drift, where the resistance drift slope $\nu$ in the $\log -\log$ plot increases with $T$. For intermediate $T$ (Fig. 7c and d), resistance drift is followed by decay, which takes place at increasingly short time for increasing $T$. Finally, at high $T$ (Fig. 7e), only resistance decay is evidenced in the considered 


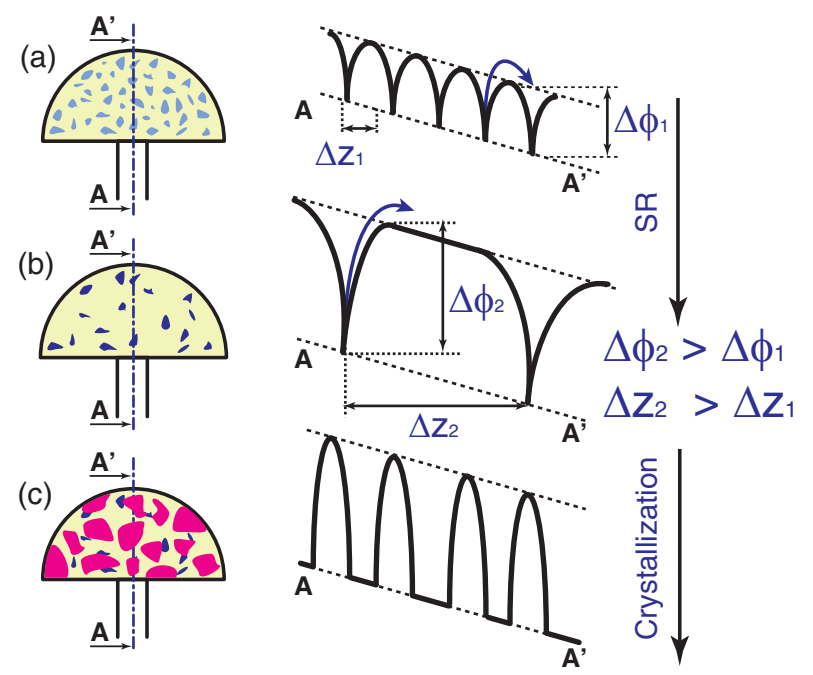

Fig. 8. Physical interpretation of the $R$ drift/decay evolution in Fig. 6a for the reset state. Conduction in the amorphous phase is driven by hopping between energy barrier of height $E_{C}$ (a). Structural relaxation (SR) consists of the annealing of defects, which leads to an increase of $E_{C}$ (b), followed by the nucleation of crystalline grains (c), causing the drop of $R$.

range of experimental times.

\section{Physical interpretation of Resistance INSTABILITY}

Fig. 8 describes the physical interpretation for resistance drift and decay in the amorphous reset state. Conduction in the amorphous phase is due to thermally-activated hopping among localized states in the mobility gap [20]. The resistance can thus be analytically described by a Poole-Frenkel (PF) equation given by:

$$
R=R_{0} e^{\frac{E_{C}}{k_{B} T}}
$$

where $R_{0}$ is a pre-exponential constant and $E_{C}$ is the effective (average) energy barrier for hopping (Fig. 8a). Drift is attributed to structural relaxation (SR) of the non-equilibrium amorphous state [21], resulting in an annealing of the localized states and in a consequent increase of the PF barrier height $E_{C}$ (Fig. 8b). The barrier increase leads to an increase of $R$ in Eq. (2), thus accounting for the observed resistance drift. Crystallization then follows by nucleation and growth of crystalline grains, resulting in a decrease of the number and heights of energy barriers and a consequent decrease of $R$ (Fig. 8c).

Following the explanation of reset-state drift and crystallization in Fig. 8, we interpreted the set-state resistance instability by SR and crystallization of residual disordered phase in Ge-rich Ge-Sb-Te in Fig. 9. The residual disordered regions might be localized, for instance, at the grain boundary (GB) between crystalline grains, as indicated in Fig. 9a, or in some confined region along the conduction path. Previous literature works [22], [23] showed how conduction in polycrystalline semiconductors appears limited by the GBs, acting as high-gap semiconductors due to the segregated defects and local disorder. Following this picture, the PF equation in our model was used to describe thermal emission over the barrier, rather than the PF conduction in the residual amorphous phase

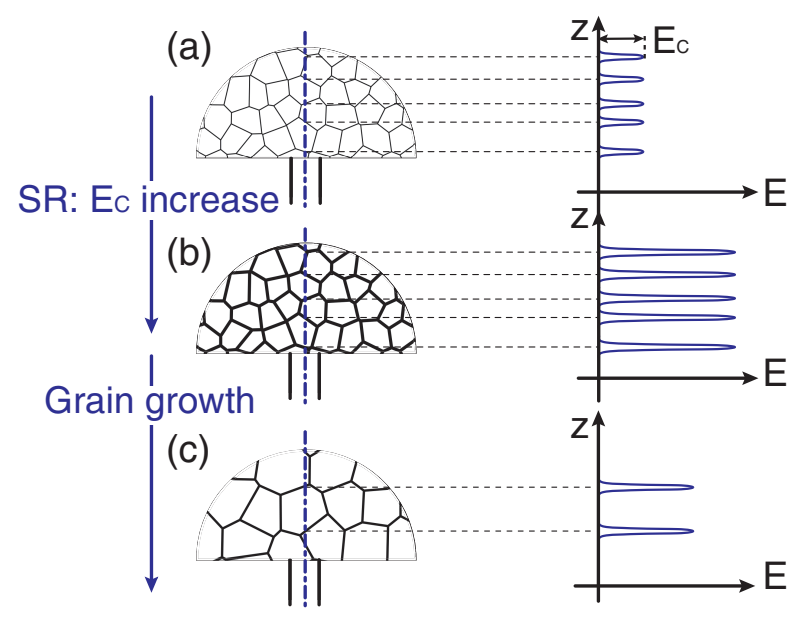

Fig. 9. Physical interpretation of the $R(t)$ behavior of the set state in Fig. 6b. Conduction is controlled by the residual amorphous phase at grain boundaries (GB). SR at GBs causes an increase of $E_{C}$ (drift), while grain growth leads to $E_{C}$ reduction and $R$ decay.

itself. Thus, $R$ in the set state can be expressed similar to Eq. (2) with proper choice of $E_{C}$. To support this conduction model, Fig. 10 shows the measured $R$ and $E_{C}$ for PCM with alloy $\mathrm{A}$ in the set state, before and after annealing at various temperature and time. For all states, $E_{C}$ shows a value of few $\mathrm{meV}$, thus consistent with the presence of a conduction barrier controlling the resistance, as shown in Fig. 9a. SR of the residual amorphous phase causes an increase of $E_{C}$ (Fig. 9b) according to Eq. (2), thus leading to $T$-accelerated drift of the set state, evidenced in Fig. 7b. This physical interpretation is supported by results in Fig. 10, showing an increase of $R$ and of $E_{C}$ after moderate annealing of $1 \mathrm{~h}$ at $150^{\circ} \mathrm{C}$ in the set state. Further annealing results in grain growth and crystallization of the residual amorphous phase, resulting in a decrease of the height and number of energy barriers along the current flow (Fig. 9c) and to a consequent decay of $R$, as evidenced in Fig. 6b. This is demonstrated by the decay of $R$ and $E_{C}$ in Fig. 10 for extensive annealing, namely 1 day at $200^{\circ} \mathrm{C}$ and $230^{\circ} \mathrm{C}$. Note the good correlation between $R$ and $E_{C}$ in both the drift and the decay regimes of the set state, which support the use of Eq. (2) for the set state and the physical interpretation of set-state resistance instability in Fig. 9. Our physical interpretation is also able to explain why set resistance drift/decay was not reported for conventional GST cells: The faster crystallization of GST would result in larger crystalline grains, with no or eventually few GBs along the conduction path.

\section{Set-State Reliability Modeling}

The physical interpretation in Fig. 9 and the experimental results in Fig. 10 provide the basis for our model of the resistance instability in the set state. SR was described as $T$-accelerated annealing of defect, where each defect in the mobility gap displays a characteristic energy barrier $E_{S R}$ for thermally-accelerated defect annealing [21]. The energy distribution $g_{S R}\left(E_{S R}\right)$ of defect states thus evolves with time 


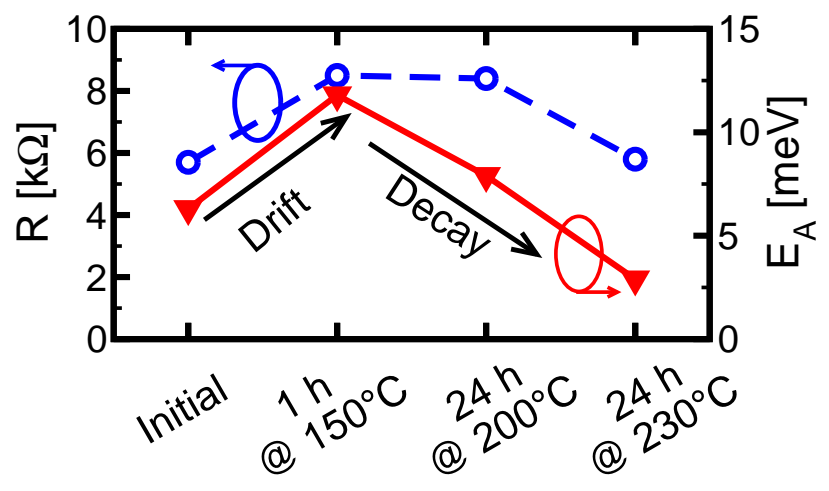

Fig. 10. Measured $R$ and activation energy $E_{C}$ for conduction of the set state at various annealing conditions. These results allow attributing the drift/decay effects to $E_{C}$, see Eq. (5).

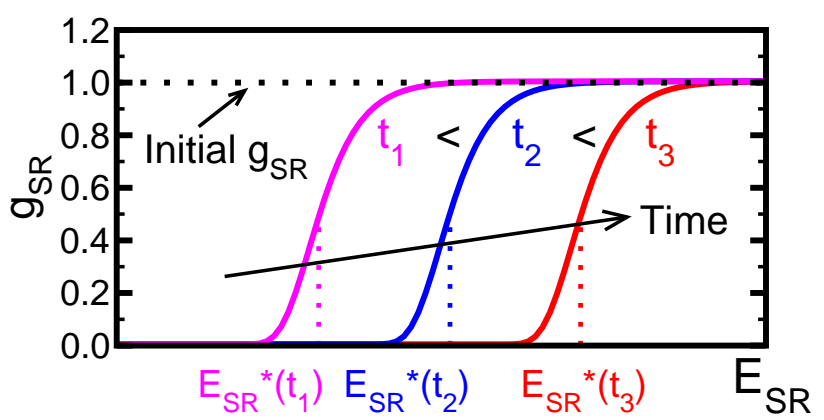

Fig. 11. Calculated defect distribution $g$ as a function of $E_{S R}$ at increasing time for $T=150^{\circ} \mathrm{C}$. The initial distribution was assumed constant, then defects are annealed moving from low to high energy $E_{S R}$.

according to the rate equation [21]:

$$
\frac{d g_{S R}}{d t}=-\frac{g_{S R}}{\tau_{0}} e^{-\frac{E_{S R}}{k_{B} T}}
$$

where $\tau_{0}$ depends on $E_{S R}$ through the Meyer-Neldel (MN) rule [24]:

$$
\tau_{0}=\tau_{00 S R} e^{-\frac{E_{S R}}{k_{B} T_{M N}}}
$$

where $\tau_{00 S R}=8 \mathrm{~s}$ and the Meyer-Neldel temperature $T_{M N}=$ $535 \mathrm{~K}$ is the iso-kinetic $T$ at which all defects show the same annealing time. Fig. 11 shows the calculated $g_{S R}\left(E_{S R}\right)$ at an annealing temperature of $150^{\circ} \mathrm{C}$. First, states at low $E_{S R}$ decay, since their corresponding transition time is shorter in Eq. (3). As time increases, states at larger $E_{S R}$ decay, thus the density of defect states $g_{S R}\left(E_{S R}\right)$ at any given time is virtually zero below a characteristics annealing front, defined by the energy $E_{S R}^{*}$ for which $g_{S R}=0.5$. Fig. 11 indicates that the annealing front energy $E_{S R}^{*}$ increases for increasing time.

The SR kinetics in the set state can be furthered analyzed from Fig. 12a, showing the Arrhenius plot of the measured and calculated $t_{S R}$, defined as the time for $R$ to reach a given resistance level, namely $1.3 R_{0}, 1.4 R_{0}$ or $1.5 R_{0}$ in the drift regime at constant $T$ (see Fig. 7a). Since $R$ identifies the annealed state, the activation energy obtained in the Arrhenius plot corresponds to the energy $E_{S R}^{*}$ describing the same state in Fig. 11 [21]. The energy $E_{S R}^{*}$ is reported in Fig. $12 \mathrm{~b}$ as a function of $R / R_{0}$ describing the annealed state: $E_{S R}^{*}$ increases for increasing $R$, since a larger $R$ corresponds to stronger

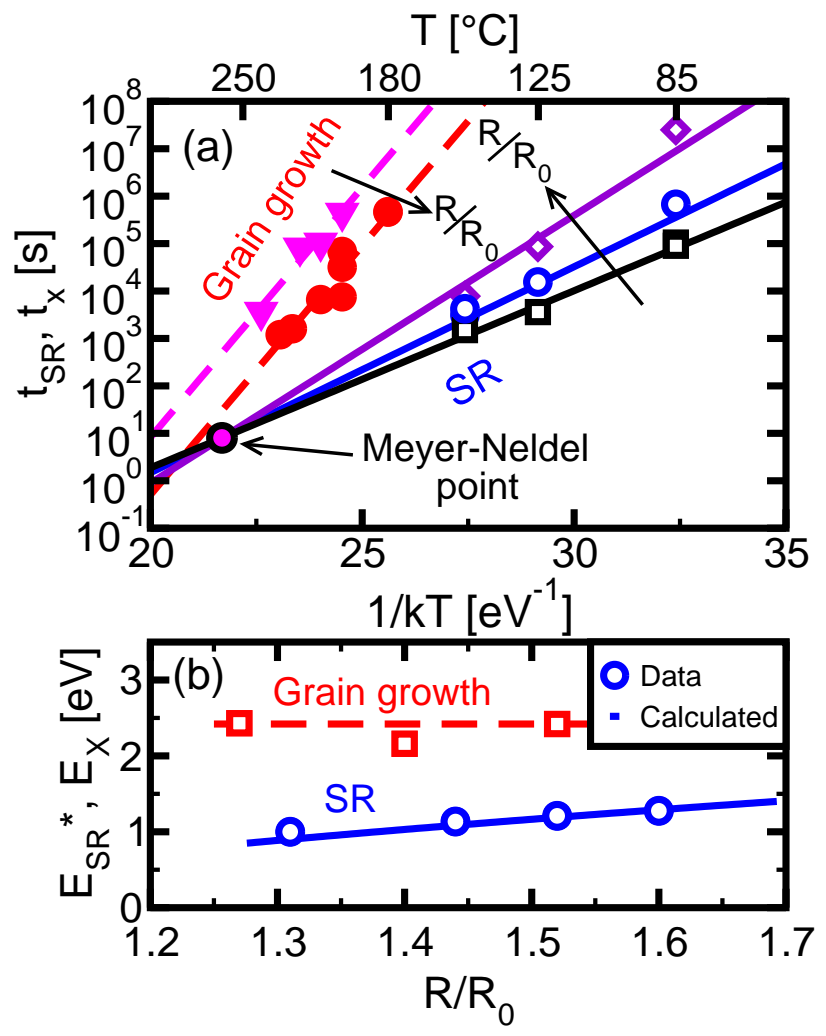

Fig. 12. (a) Measured and calculated Arrhenius plot of $t_{S R}$ and $t_{X}$ for variable threshold $R / R_{0}$ in Fig. 7 and (b) extracted activation energies for SR $\left(E_{S R}\right)$ and grain growth $\left(E_{X}\right)$ as a function of $R / R_{0}$. While $E_{S R}^{*}$ increases during annealing, $E_{X}$ is almost constant.

relaxation, namely more extensive annealing of defects in Fig. 11. Fig. 13a shows the calculated $E_{S R}^{*}$ as a function of time for $T=150^{\circ} \mathrm{C}$, evidencing the shift of the annealing front at increasing time.

Fig. 12a also reports the Arrhenius plot of $t_{X}$, defined as the time to reach a value $R$ in the decay region (see Fig. 7d). The slope of $t_{X}$ in the Arrhenius plot yields $E_{X}$, namely the energy barrier for crystallization of the residual amorphous phase in the poly-crystalline state. Fig. 12b shows that $E_{X}$ remains almost constant for all values of $R$. To describe crystallization, a rate equation similar to Eq. (3) was thus adopted, where the activation energy for crystallization was kept constant at $E_{X}=2.42 \mathrm{eV}$ and the pre-exponential time $\tau_{0}$ is distributed in the amorphous material by a density function $g_{X}\left(\tau_{0}\right)$. In a similar way as before, it is possible to describe the crystallization front by $\tau_{0}^{*}$ taken at $g_{X}=0.5$. Fig. 13a also shows the calculated $\tau_{0}^{*}$ as a function of time for $T=150^{\circ} \mathrm{C}$ : As $\tau_{0}^{*}$ becomes comparable to a critical value $\tau_{0 X}^{*}=3 \times 10^{-23} \mathrm{~s}$, crystallization takes place. Tab. I summarizes the main parameters used in the model for various PCM states. Fig. 12a also shows calculation results for $t_{S R}$ and $t_{X}$, while Fig. 12b shows the calculated $E_{S R}^{*}$ as a function of $R / R_{0}$. The good agreement in both cases supports the accuracy of our model for SR and crystallization.

To describe the impact of SR and crystallization processes on $R$, the activation energy $E_{C}$ of resistance is the modeled as:

$$
E_{C}=\alpha E_{S R}^{*}\left(1-\beta \tanh \frac{\log \tau_{0}^{*}-\log \tau_{0 X}^{*}}{\eta}\right)
$$




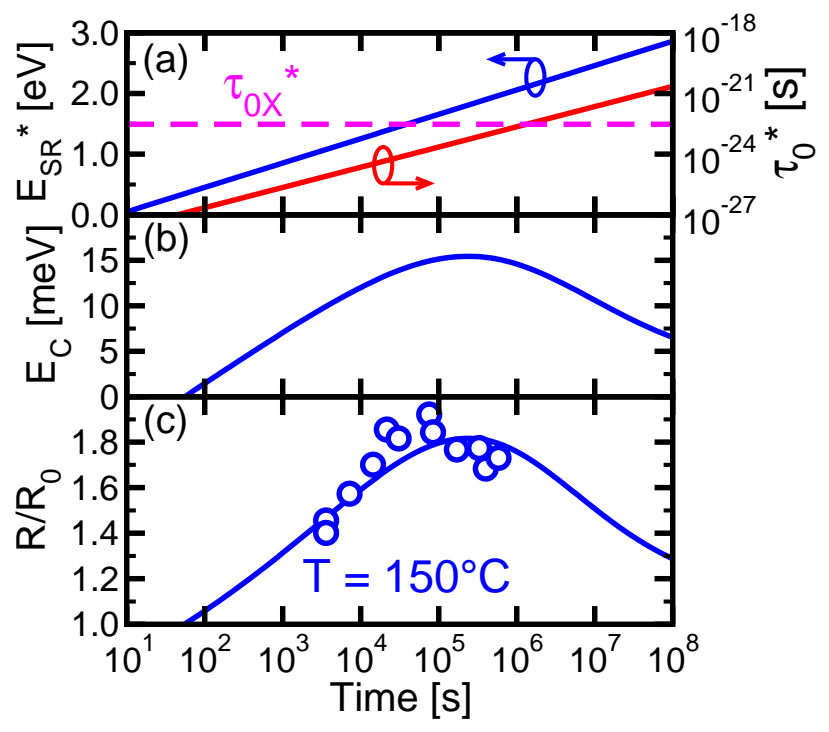

Fig. 13. (a) Calculated evolution of $E_{S R}^{*}$ (left) and $\tau_{0}^{*}$ (right) by Eq. (3) $\left(\tau_{0 X}^{*}\right.$ is shown for reference) at $T=150^{\circ} \mathrm{C}$, (b) calculated $E_{C}$ for conduction by Eq. (5) and (c) measured/calculated $R / R_{0}$ by Eq. (2).

For $\tau_{0}^{*} \ll \tau_{0 X}^{*}$ (drift regime), Eq. (5) predicts that $E_{C}$ increases linearly with $E_{S R}^{*}$ according to a parameter $\alpha=0.014$, thus describing resistance drift through Eq. (2). As $\tau_{0}^{*}$ becomes comparable to $\tau_{0 X}^{*}$ (crystallization, or grain growth regime), the second term in Eq. (5) results in a gradual decrease of $E_{C}$, where $\beta=0.9$ controls the resistance decrease upon decay and $\eta=4.3$ determines the slope of the decay, thus accounting for crystallization. Fig. $13 \mathrm{~b}$ shows the calculated $E_{C}$, obtained by Eq. (5), evidencing the drift regime, where $E_{C}$ increases linearly with $E_{S R}^{*}$, followed by the crystallization regime, where $E_{C}$ decays as $\tau_{0}^{*}$ approaches the critical value $\tau_{0 X}^{*}$.

The resistance is then obtained from $E_{C}$ through Eq. (2), as shown in Fig. $13 \mathrm{c}$ for $T=150^{\circ} \mathrm{C}$. Calculations were repeated at different annealing temperatures under isothermal conditions, as shown in Fig. 7: In all cases, a very good agreement is obtained in both the drift and the decay regimes. The drift regime can be described by the slope $\nu$ in the log-log plot. The crossing from drift to crystallization is instead marked by the maximum of resistance $R_{\max }$, occurring at a characteristic time $t_{\max }$ (see definition in Fig. 7c, $\mathrm{d}$ and e). These parameters determine the evolution of resistance window during memory operation, thus they play an essential role in reliability control and prediction of ePCM devices.

Fig. 14 shows the Arrhenius plot of measured and calculated $R_{\max }$ (a), $t_{\max }$ (b) and $\nu$ (c) for the set state during isothermal conditions. The transition time $t_{\max }$ decreases with $T$, as

TABLE I

ARRHENIUS AND MN PARAMETERS FOR SIMULATING THE KINETICS OF SR AND CRYSTALLIZATION/GRAIN GROWTH FOR THE SET, THE INCOMPLETE SET AND THE RESET STATES.

\begin{tabular}{lcccc}
\hline \hline & $\tau_{00 S R}[s]$ & $\tau_{0 X}^{*}[s]$ & $E_{X}[e V]$ & $T_{M N}[K]$ \\
\hline Set & 8 & $3 \cdot 10^{-23}$ & 2.42 & 535 \\
Incomplete set & 8 & $3 \cdot 10^{-23}$ & 2.60 & 535 \\
Reset & 10 & $3 \cdot 10^{-39}$ & 4.20 & 550 \\
\hline
\end{tabular}

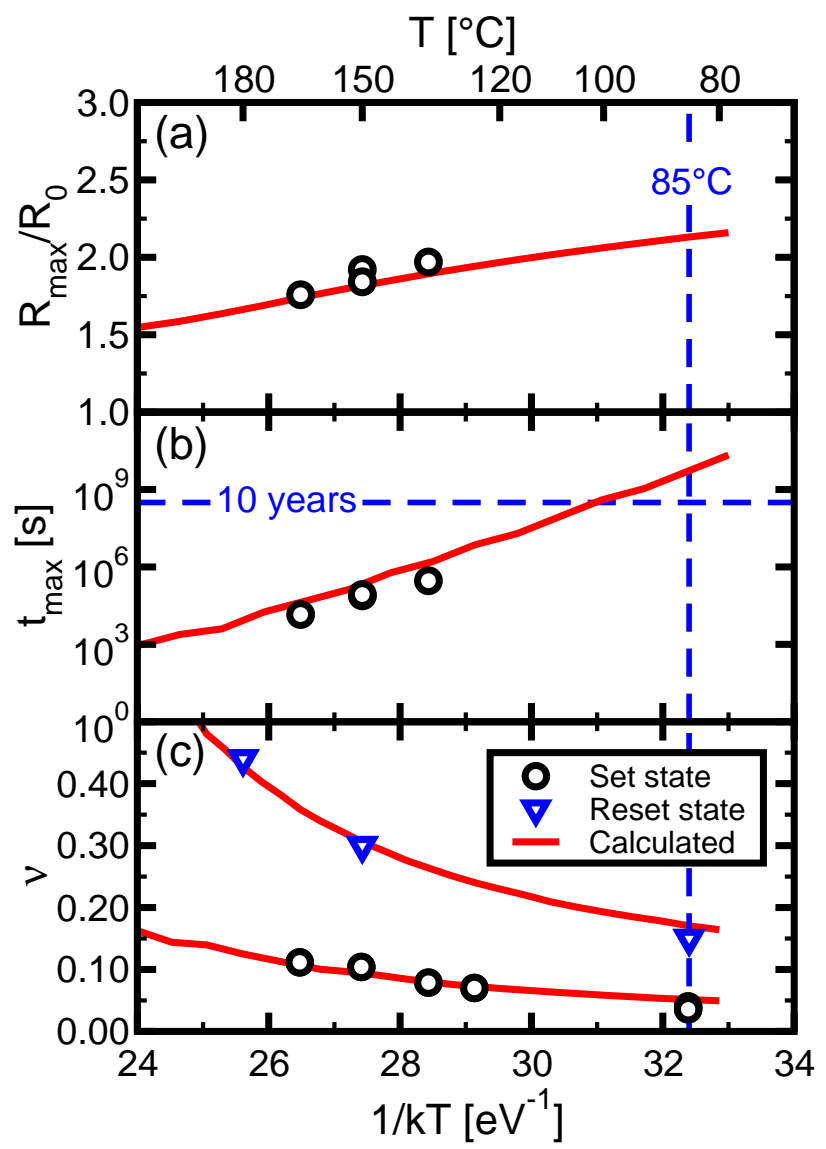

Fig. 14. Arrhenius plot of the measured and calculated maximum resistance $R_{\max }$ (a), the corresponding time $t_{\max }$ (b) and the drift exponent $\nu$ of set and reset states (c). The decrease of $t_{\max }$ (b) and the increase of $\nu$ (c) with $T$ are due to the thermal activation of crystallization and SR respectively, while the decrease of $R_{\max }$ with $T$ (a) is explained in Fig. 15.

a result of the temperature-acceleration of the crystallization processes. Similarly, the increase of $\nu$ with $T$ can be explained by the $T$-acceleration of SR. The $T$-dependence of $R_{\max }$ can instead be understood by considering the Arrhenius plot of SR and crystallization processes in Fig. 12. To this purpose, Fig. 15 schematically shows the Arrhenius plot of $t_{S R}$ and $t_{X}$, the latter evaluated at the onset of the decay regime at $R_{\max }$. Two isothermal annealing are compared at temperatures $T_{1}$ and $T_{2}$ with $T_{1}>T_{2}$. For $T=T_{1}$, the crystallization time $t_{X}$ crosses $t_{S R 1}$, corresponding to a resistance value $R_{\max 1}$ which thus marks the transition between drift and decay. For $T=T_{2}<T_{1}$, instead, $t_{X}$ crosses a different SR time $t_{S R 2}$ with $E_{S R 2}^{*}>E_{S R 1}^{*}$, thus corresponding to a more relaxed state with larger resistance $R_{\max 2}$. Since $R_{\max 2}>R_{\max 1}$, the maximum resistance at the crossing between drift and decay increases for decreasing temperature in a isothermal annealing experiment. Note that this result is due to the crystallization time $t_{X}$ passing below the isokinetic point where all $t_{S R}$ line cross each other. This interpretation allows to explain the results in Fig. 14a, which highlights that low-temperature annealing, while causing less acceleration of drift, results in a larger reduction of the available read window at $t_{\max }$, which should be taken into account for reliability prediction. 


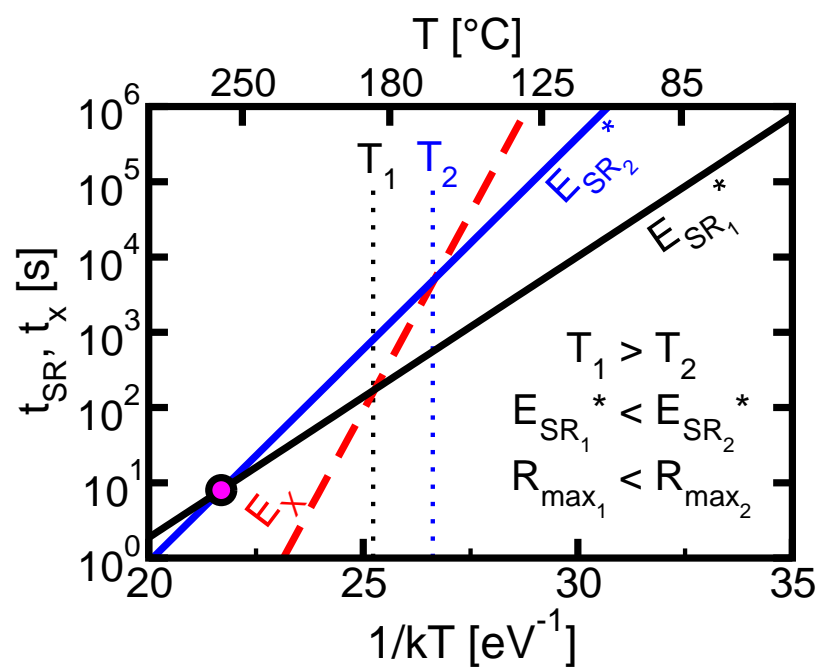

Fig. 15. Simplified sketch of the Arrhenius plot of SR and crystallization time in Fig. 12. The crystallization front at the transition between the drift and the decay regimes (dotted line) crosses two different SR fronts at $T_{1}$ and $T_{2}$ (with $T_{1}>T_{2}$ ). Note that $E_{S R 1}^{*}<E_{S R 2}^{*}$. Since $E_{C}$ increases with $E_{S R}^{*}$ (Eq. (5)), this explains why $R_{\max }$ in Fig. 14a decreases at increasing

\section{UNIFIED MODEL}

The experimental results in Fig. 6 demonstrates that set and reset states display similar annealing behavior with resistance drift followed by resistance decay. Therefore, the model for set-state resistance evolution was extended to the reset state and to intermediate states in PCM with alloy A as active chalcogenide material. The reset state is characterized by a larger activation energy $E_{X}=4.3 \mathrm{eV}$ (Fig. 3): This is markedly larger than $E_{X}=2.3 \mathrm{eV}$ in the set state, possibly because the amorphous region extends to the whole programmed dome in the reset state. On the other hand, the residual amorphous phase at GBs in the set state might suffer from easy crystallization due to the adjacent crystalline grains. The incomplete set state, obtained by a triangular set pulse with $t_{q}=1 \mu \mathrm{s}$, displayed activation energy $E_{X}=2.6 \mathrm{eV}$ intermediate between the set and the reset states possibly due to the larger residual amorphous phase with thicker GBs and smaller grains [25]. Model parameters used in our calculations for the reset and intermediate states are reported in Tab. I. Calculation results by the unified model are shown in Fig. 6a (reset and intermediate states) and Fig. 6b (set state) for $R$ measured after annealing at fixed time $(1 \mathrm{~h})$ and increasing $T$. The measured and calculated slope of resistance drift for the reset state is also shown in Fig. 14c to further support the accuracy of the unified model.

Fig. 16a shows the measured and calculated $R$ as a function of time for the set and reset states at $T=85^{\circ} \mathrm{C}$ and $150^{\circ} \mathrm{C}$, to describe possible worst cases in consumer and automotive applications, respectively. For the sake of clarity, Fig. 16b shows results for the set state on a smaller resistance scale. Calculation results closely reproduce data for experimental times below $10^{6} \mathrm{~s}$ and allow prediction of the resistance evolution at longer times. According to the model, data retention is guaranteed up to 10 years, with a minimum resistance window in correspondence of $t_{\max }$ of more than 2 decades, assuming a minimum reset-state resistance of $1 \mathrm{M} \Omega$ for shorter times

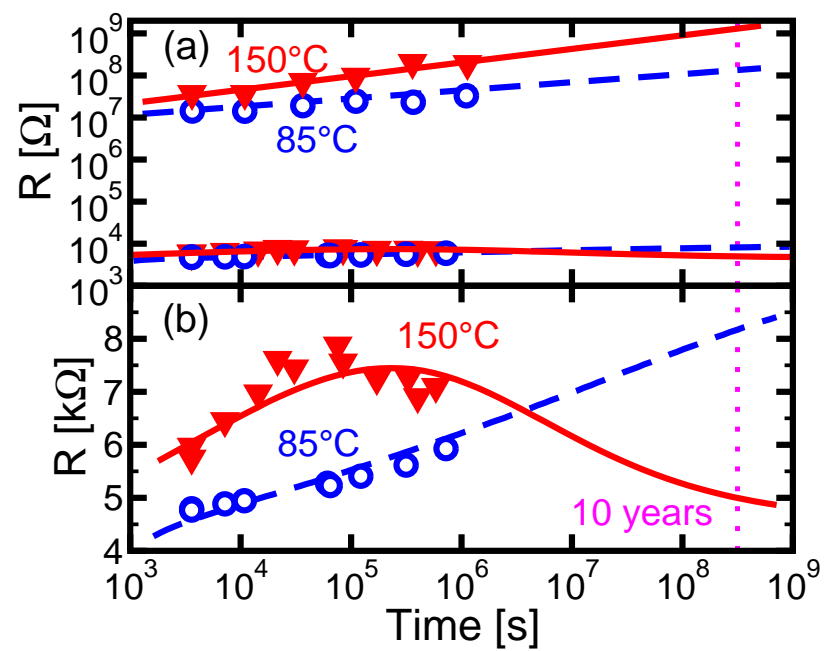

Fig. 16. (a) Measured and calculated $R$ for set/reset states during isothermal annealing at $85^{\circ} \mathrm{C}$ and $150^{\circ} \mathrm{C}$. The evolution of the set state is highlighted in (b).

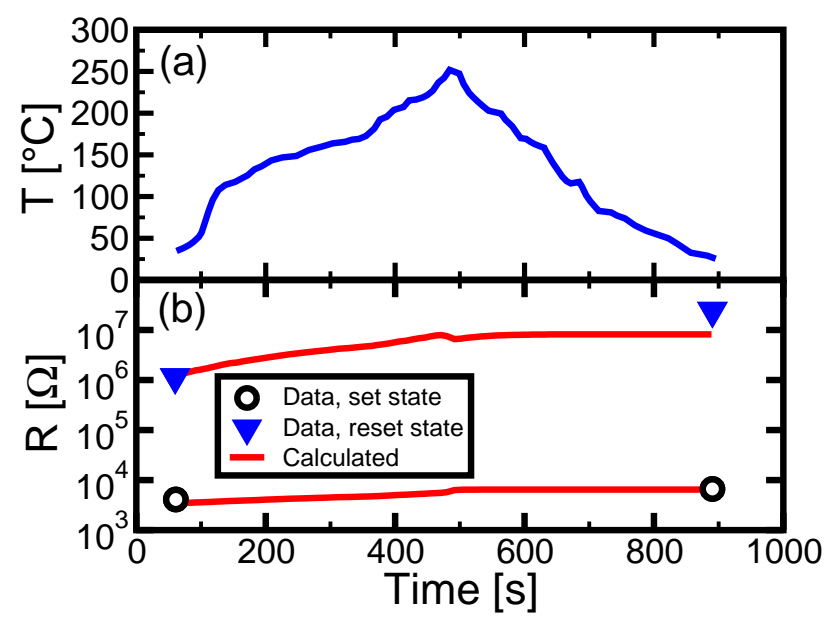

Fig. 17. Temperature evolution during soldering according to the JEDEC standard [26] (a) and corresponding calculated evolution of $R$ for the set and reset states (b). Data and calculations confirm good data retention after soldering reflow process.

not shown in the figure. The set state displays pure drift at $85^{\circ} \mathrm{C}$, since $t_{\max }$ is larger than 10 years in this case. On the other hand, $t_{\max }$ is around $10^{5} \mathrm{~s}$ for $150^{\circ} \mathrm{C}$, with a maximum resistance of about $8 \mathrm{k} \Omega$. The anticipated decay has a beneficial impact on the resistance window, due to the $T$-dependence of $R_{\max }$ in Fig. 16a.

Finally, we investigated data retention upon soldering reflow process [12], [27]. Fig. 17a shows the soldering temperature profile according to the JEDEC standard [26], reaching a maximum temperature of $250^{\circ} \mathrm{C}$. Fig. $17 \mathrm{~b}$ shows the measured and calculated resistance before/after soldering. Data were obtained from the median value within 100 cells, while the calculations were performed by Eq. (3) using the time evolution of $T$ in Fig. 17a. The model is able to predict the resistance evolution for both set and reset states even in non-isothermal conditions. Experiments and calculations demonstrate data retention upon soldering reflow process. 


\section{CONCLUSIONS}

Embedded PCM offers several advantages in terms of cost and scalability compared to Flash memory, however the tight reliability requirements must be met through material engineering. The resistance instability has been analyzed in ePCM devices based on Ge-rich GeSbTe. Although Ge enrichment leads to higher crystallization point in the reset (amorphous) state, the set state displays drift and decay of resistance. These effects are interpreted by SR and crystallization of residual amorphous phase, possibly located at the GB of the poly-crystalline phase. A new model is developed to describe SR and crystallization in the set state, then extended to the reset and incomplete set states to achieve a unified model of resistance change with time. The model allows for physics-base extrapolation of resistance variation, allowing to demonstrate data retention at $85^{\circ} \mathrm{C}$ and $150^{\circ} \mathrm{C}$ for 10 years and after soldering reflow.

\section{REFERENCES}

[1] D. Loke, T. H. Lee, W. J. Wang, L. P. Shi, R. Zhao, Y.-C. Yeo, T.-C. Chong, and S. R. Elliott, "Breaking the speed limits of phase-change memory," Science, vol. 336, pp. 1566-1569, 2012.

[2] F. Xiong, A. D. Liao, D. Estrada, and E. Pop, "Low-power switching of phase-change materials with carbon nanotube electrodes," Science, vol. 332, pp. 568-570, 2011.

[3] G. Servalli, "A 45nm generation phase change memory technology," in IEDM Tech. Dig., 2009, pp. 113-116.

[4] H. Y. Cheng, M. BrightSky, S. Raoux, C. F. Chen, P. Y. Du, J. Y. Wu, Y. Y. Lin, T. H. Hsu, Y. Zhu, S. Kim, C. M. Lin, A. Ray, H. L. Lung, and C. Lam, "Atomic-level enginereeing of phase change material for novel fast-switching and high-endurance PCM for storage class memory application," in IEDM Tech. Dig., 2013, pp. 758-761.

[5] P. Zuliani, E. Varesi, E. Palumbo, M. Borghi, I. Tortorelli, D. Erbetta, G. Libera, N. Pessina, A. Gandolfo, C. Prelini, L. Ravazzi, and R. Annunziata, "Overcoming temperature limitations in phase change memories with optimized $\mathrm{Ge}_{x} \mathrm{Sb}_{y} \mathrm{Te}_{z}$," IEEE Trans. Electron Devices, vol. 60, pp. 4020-4025, 2013.

[6] H. Y. Cheng, T. H. Hsu, S. Raoux, J. Wu, P. Y. Du, M. Breitwisch, Y. Zhu, E. K. Lai, E. Joseph, S. Mittal, R. Cheek, A. Schrott, S. C. Lai, H. L. Lung, and C.Lam, "A high performance phase change memory with fast switching speed and high temperature retention by engineering the $\mathrm{Ge}_{x} \mathrm{Sb}_{y} \mathrm{Te}_{z}$ phase change material," in IEDM Tech. Dig., vol. 51, 2011, pp. 51-54.

[7] H. Y. Cheng, J. Y. Wu, R. Cheek, S. Raoux, M. BrightSky, D. Garbin, S. Kim, T. H. Hsu, Y. Zhu, E. K. Lai, E. Joseph, A. Schrott, S. C. Lai, A. Ray, H. L. Lung, and C. Lam, "A thermally robust phase change memory by engineering the $\mathrm{Ge} / \mathrm{N}$ concentration in $(\mathrm{Ge}, \mathrm{N})_{x} \mathrm{Sb}_{y} \mathrm{Te}_{z}$ phase change material," in IEDM Tech. Dig., 2012, pp. 725-728.

[8] N. Ciocchini, E. Palumbo, M. Borghi, P. Zuliani, R. Annunziata, and D. Ielmini, "Unified reliability modeling of Ge-rich phase change memory for embedded applications," IEDM Tech. Dig., pp. 581-584, 2013.

[9] G. Betti Beneventi, E. Gourvest, A. Fantini, L. Perniola, V. Sousa, S. Maitrejean, J. Bastien, A. Bastard, A. Fargeix, B. Hyot, C. Jahan, J. Nodin, A. Persico, D. Blachier, A. Toffoli, S. Loubriat, A. Roule, S. Lhostis, H. Feldis, G. Reimbold, T. Billon, B. D. Salvo, L. Larcher, P. Pavan, D. Bensahel, P. Mazoyer, R. Annunziata, , and F. Boulanger, "On carbon doping to improve GeTe-based phase-change memory data retention at high temperature," in International Memory Workshop, 2010, pp. 21-24.

[10] G. Navarro, A. Persico, E. Henaff, F. Aussenac, P. Noe, C. Jahan, L. Perniola, V. Sousa, E. Vianello, and B. D. Salvo, "Electrical performances of $\mathrm{SiO}_{2}$-Doped GeTe for phase-change memory applications," in Proc. IRPS, 2013, pp. MY 9.1-9.5.

[11] J. Akola and R. Jones, "Structural phase transitions on the nanoscale: The crucial pattern in the phase-change materials $\mathrm{Ge}_{2} \mathrm{Sb}_{2} \mathrm{Te}_{5}$ and GeTe," Phys. Rev. B, vol. 76, no. 23, pp. 235 201-1-235 201-10, 2007.
[12] G. Navarro, M. Coue', A. Kiouseloglou, P. Noe', F. Fillot, V. Delaye, A. Persico, A. Roule, M. Bernard, C. Sabbione, D. Blachier, V. Sousa, L. Perniola, S. Maitrejean, A. Cabrini, G. Torelli, P. Zuliani, R. Annunziata, E. Palumbo, M. Borghi, G. Reimbold, and B. D. Salvo, "Tradeoff between set and data retention performance thanks to innovative materials for phase-change memory," in IEDM Tech. Dig., 2013, pp. 570-573.

[13] A. Pirovano, A. Redaelli, F. Pellizzer, F. Ottogalli, M. Tosi, D. Ielmini, A. L. Lacaita, and R. Bez, "Reliability study of phase change nonvolatile memories," IEEE Trans. Device Mater. Reliab., vol. 4, pp. 422-427, 2004.

[14] D. Mantegazza, D. Ielmini, E. Varesi, A. Pirovano, and A. L. Lacaita, "Statistical analysis and modeling of programming and retention in PCM arrays," in IEDM Tech. Dig., 2007, pp. 311-314.

[15] iG. Novielli, A. Ghetti, E. Varesi, A. Mauri, and R. Sacco, "Atomic migration in phase change materials," in IEDM Tech. Dig., 2013, pp. 589-592.

[16] M. Boniardi and D. Ielmini, "Physical origin of the resistance drift in amorphous phase-change materials," Appl. Phys. Lett., vol. 98, p. 243506, 2011.

[17] N. Ciocchini, M. Cassinerio, D. Fugazza, and D. Ielmini, "Modeling of threshold-voltage drift in phase-change memory (PCM) devices," IEEE Trans. Electron Devices, vol. 59, pp. 3084-3090, November 2012.

[18] D. Ielmini, A. L. Lacaita, and D. Mantegazza, "Recovery and drift dynamics of resistance and threshold voltages in phase-change memories," IEEE Trans. Electron Devices, vol. 54, pp. 308-315, 2007.

[19] J. Li, T. H. B. Luan, Y. Zhu, G. Martyna, D. Newns, H. Cheng, S. Raoux, H. L. Lung, and C. Lam, "Explore physical origins of resistance drift in phase change memory and its implication for drift-insensitive materials," in IEDM Tech. Dig., 2011, pp. 291-294.

[20] D. Ielmini and Y. Zhang, "Analytical model for subthreshold conduction and threshold switching in chalcogenide-based memory devices," $J$. Appl. Phys., vol. 102, p. 054517, 2007.

[21] D. Ielmini, S. Lavizzari, D. Sharma, and A. L. Lacaita, "Temperature acceleration of structural relaxation in amorphous $\mathrm{Ge}_{2} \mathrm{Sb}_{2} \mathrm{Te}_{5}, " A p p l$. Phys. Lett., vol. 92, p. 193511, 2008.

[22] M. M. Mandurah, K. C. Saraswat, and T. I. Kamins, "A model for conduction in polycrystalline silicon-part I: Theory," IEEE Trans. Electron Devices, vol. 28, pp. 1163-1171, 1981.

[23] C. T. Angelis, C. A. Dimitriadis, I. Samaras, J. Brini, G. Kamarinos, V. K. Gueorguiev, and T. E. Ivanov, "Study of leakage current in nchannel and p-channel polycrystalline silicon thin-film transistors by conduction and low frequency noise measurements," J. Appl. Phys., vol. 82, pp. 4095-4101, 1997.

[24] D. Ielmini, M. Boniardi, A. L. Lacaita, A.Redaelli, and A. Pirovano, "Unified mechanisms for structural relaxation and crystallization in phase-change memory devices," Microelectron. Eng., vol. 86, pp. 19421945, 2009.

[25] G. W. Burr, P. Tchoulfian, T. Topuria, C. Nyffeler, K. V., A. Padilla, R. M. Shelby, M. Eskandari, B. Jackson, and B.-S. Lee, "Observation and modeling of polycrystalline grain formation in $\mathrm{Ge}_{2} \mathrm{Sb}_{2} \mathrm{Te}_{5}$," J. Appl. Phys., vol. 111, p. 104308, 2012.

[26] Moisture/Reflow Sensitivity Classification for Nonhermetic Solid State Surface Mount Devices, IPC/JEDEC J-STD-020C, July 2004.

[27] H. Lung, M. Breitwisch, J. Wu, P.-Y. Du, Y. Zhu, M. Lee, Y. Shih, E. K. Lai, R. Dasaka, T. Wang, C. Chen, R. Cheek, A. Schrott, E. Joseph, H. Cheng, S. Raoux, and C. Lam, "A method to maintain Phase-Change Memory pre-coding data retention after high temperature solder bonding process in embedded systems," in VLSI Tech. Dig., vol. 98, 2011. 